\title{
La alzada del caballo antiguo: un estado de la cuestión aplicado a la Edad del Hierro de la Península Ibérica.
}

\author{
Fernando Quesada Sanz
}

Universidad Autónoma de Madrid

\section{Resumen}

Aunque existían variaciones regionales importantes, la alzada de todos los caballos de la Antigüedad era por lo general inferior a lo que en la actualidad marca el límite entre caballos y poneis. Sin embargo, el reducido tamaño de los caballos no limita su capacidad para el tiro o la monta: muchos ponis bien conformados son tan resistentes o más que los caballos. En este artículo se presenta un estado de la cuestión sobre el tamaño de los caballos antiguos en Eurasia entre el comienzo de la Edad del Hierro y el comienzo de la Alta Edad Media, y se recoge por primera vez un cuadro con las mediciones disponibles efectuadas sobre restos arqueozoológicos propios del ámbio ibérico en la Edad del Hierro, de donde se deduce que el panorama de dimensiones en lberia concuerda básicamente con el de el resto de Europa Occidental

Palabras Clave: Caballos. Alzada. Edad del Hierro. Península Ibérica. Siglos VII-I a.C.

\section{Summary}

Although there were clear regional groups, the size (height at the withers) of horses in Antiquity was smaller than the modern divide between horses and poneys, i.e. c. $148 \mathrm{~cm}$. However, a horse's small size does not limit significantly its capacity as a draught, pack or riding animal: a well proportioned pony can be as enduring as a horse. This paper presents a short overview of the current state of research on horse size in Eurasia from the First Iron Age to the Late Roman Empire and the Dark Ages, and for the first time summarizes the available evidence on horse size during the Iberian Iron Age (7th to 1st centuries BC), showing a pattern that closely resembles that of Central and Western Europe.

Keywords: Horses. Height at the withers. Iron Age. Iberian Peninsula. 7th-1st centuries BC

"The size of the horse does not have as great a bearing on its ability to carry weight as would at first appear, but its conformation does, and this also affects its durability. Speed is not a major requirement for a warhorse". Hyland (1990:67).

El propósito de este trabajo es limitado: trataremos de recopilar los datos analíticos hasta ahora publicados sobre la alzada de los équidos de la

1 Trabajo realizado en el marco del Proyecto de Investigación "La imagen de las armas en la Iberia prerromana" (BHA 2001-0187) Agradecemos a la Dra. Corina Liesau von Lettow-Vorbeck sus sugerencias y
Edad del Hierro en la Península Ibérica, insertándolos dentro del marco general de los estudios sobre el tamaño de los caballos en Europa

observaciones sobre una primera versión del manuscrito, aunque lógicamente no es responsable de posibles errores u omisiones, ni de las opiniones vertidas en el trabajo. 
Occidental en el mismo periodo. Aún dentro de sus limitaciones, la publicación de esta primera síntesis a partir de datos hasta ahora dispersos en numerosos informes arqueozoológicos y algunos trabajos más generales de especialistas en análisis de fauna, entre los que no pretendemos en ningún caso contarnos, constituye una buena base de partida para estudios ulteriores y plantea la necesidad de un estudio en equipo más sistemático de los datos disponibles con amplias miras tanto en lo geográfico como en lo cronológico. Por otra parte, incluso con las limitaciones que a continuación detallaremos, los datos reunidos son ya útiles para estudios ulteriores sobre el empleo militar y económico de los équidos en la Iberia prerromana.

El principal problema de los datos peninsulares es la escasez de équidos en los yacimientos, que limita el propio corpus de partida, sobre todo en comparación con la abundancia comparativa de información arqueológica en la Galia o Germania. En segundo lugar, la información publicada es muy heterogénea: bastantes informes son ya añejos y escuetos, y no se conforman a los estándares actuales; a veces no se proporcionan algunos datos de metodología que serían necesarios (Morales 1988, 1990), y la dispersión cronológica es muy grande, entre el s. VII y el I a.C. Además, de entre los cerca de doscientos yacimientos catalogados, apenas una veintena han podido proporcionar datos de alzada. Tampoco conocemos estudios publicados de síntesis que traten sistemáticamente la cuestión del tamaño de los équidos en Iberia durante la Edad del Hierro. Por otro lado, no se abordarán aquí tres cuestiones relacionadas con la del tamaño, pero que exigirían un espacio y unos datos de los que simplemente no disponemos: la cuestión del dimorfismo sexual -en todo caso menos significativa que en otros animales como los bóvidos ( $c f$. Meniel 1996:114)-, la de las hibridaciones y la de la domesticación en Iberia, en todo caso muy anterior a la I Edad del Hierro. ${ }^{2}$ La casi totalidad de los informes publicados pasa de puntillas sobre las dos primeras cuestiones. Pese a todo, aunque sin duda existe variación de talla por sexos, ni en Iberia ni en el resto de Europa parece que la cuestión afecte al hecho básico documentado masivamente de una talla reducida en comparación con la actual. ${ }^{3}$ Esto es, aunque pudiéramos distinguir con seguri-

2 Para la cuestión de la domesticación en general, y el caso de Iberia en particular, ver las siguientes discusiones recientes -desde 1990- con referencias a la bibliografía anterior: Uerpmann (1990); Meadow, Uerpmann (eds.) (1991); Bökönyi (1993); Levine (1993); Uerpmann (1995); Eisenmann (1996); Gautier (1996); Gheorghiou (1996); Liesau (1998, 2005); Morales et al. (1996); en general los trabajos recogidos en Anreiter et al. 8eds.) (1998) y Levine, Renfrew, Boyle (2003); Levine (2004); Eisenmann (e.p.) dad en los datos hispanos yeguas de sementales -que no podemos-, sabemos que en la antigüedad se utilizaban ambos para la monta, y no parece que el dimorfismo altere el patrón general que es el que ahora nos interesa. En cuanto a las hibridaciones, la situación es similar: pese a algunos esfuerzos aislados sobre el problema de la identificación de mulas, apenas conocemos nada sobre la relación entre esa cuestión y el tema de la talla de los animales.

Para la comparativa con el resto de Europa lógicamente hemos acudido sobre todo, además de a algunos informes señeros que han orientado la investigación durante las décadas ulteriores, a trabajos de síntesis para cada periodo lo más recientes posible, tanto por parte de arqueozoólogos como de historiadores, con la simple idea de proporcionar un marco de referencia comprensible para los datos peninsulares. No intentamos siquiera, pues, un análisis detallado de los problemas de domesticación, de las tallas de los animales en la Edad del Bronce en el Próximo Oriente o Europa, de los problemas de evolución y cría que afectan al crecimiento de los animales, o de los diferentes informes detallados para cada región, porque ese no es el objetivo de la breve recopilación presentada. Sin embargo, la información presentada es a nuestro juicio representativa del estado de la cuestión, en particular en las regiones más próximas a Iberia: Francia e Italia.

Desde el punto de vista de la metodología de análisis, lógicamente no podemos pretender una revisión directa de los materiales de cada yacimiento: eso exigiría un proyecto coordindado de amplio alcance con la colaboración de arqueozoólogos e historiadores, que quizá convendría abordar pronto; tampoco podemos ser pues responsables de la metodología empleada en cada caso y su adecuación o no. Entretanto, la principal objeción que encontramos a los datos reunidos es que los diferentes análisis no son homogéneos en su metodología, empleando criterios diferentes como el elemento empleado para la determinación de altura en la cruz, o los índices multiplicadores. Por ello siempre que nos ha sido posible hemos indicado los 'recorridos' estadísticos cuando los diferentes autores los proporcionan. También hemos mantenido las cifras decimales cuando los autores las dan.

3 Por ejemplo, Meniel (1990:273) "Les chevaux son beaucoup moins marqués [que los bóvidos] par le dimorphisme sexuel, mais ils présentent une varieté de stature assez large. En fait, comme pour le boeuf, il ne semble pas que ce soit la taille des animaux qui augmente, mais plutöt de grands animaux qui s'ajoutent à la population indigène" [se refiere al yacimiento celta de Hornaing, de La Tène Final a galo-romano]. 
En todo caso, debemos recordar que, según el hueso que se emplee -o del que se disponga- para hacer las mediciones y extrapolar la alzada del caballo, las estimaciones obtenidas pueden varíar a veces sustancialmente (por ejemplo, Gabriel, Mees, Collin, 1995:115). Así, según el trabajo de Bökönyi al que remitimos para los detalles arqueofaunísticos y estadísticos (1974:246) se pueden emplear hasta siete criterios diferentes, que proporcionan oscilaciones en los resultados de en torno al 8-9\% (explicación simplificada en Meniel 1987:15; lista accesible de los factores de Kiesewalter 1888 aplicados a los diferentes huesos en Chaix, Meniel 1996:20). Aunque la comparación por yacimientos, y especialmente en el caso peninsular donde hay una o dos medidas por sitio, puede verse seriamente afectada por diferencia de metodología, una actitud en exceso crítica llevaría a negar la posibilidad misma de una comparativa hasta que se homogeneizaran procedimientos mediante un reestudio directo de todos los materiales (tema para una o varias Tesis Doctorales), lo que además no siempre sería posible por el tipo de hueso conservado. Por otro lado, y como se observará, la inmensa mayoría de trabajos españoles emplea los factores de Kiesewalter (1888), aunque su aplicación a diversos huesos, como también se verá, presenta oscilaciones para un mismo animal que pueden superar los $5 \mathrm{~cm}$. (caso de Sansol), lo que es un saludable caveat sobre la fiabilidad absoluta en rangos cortos (ver al respecto también Liesau 1998:94).

Según criterios actuales, la altura en la cruz que separa un caballo de un poni está en torno a los $142-148 \mathrm{~cm}^{4}$ Aunque tal criterio, como otros muchos, no deja de ser algo artificial, nos proporciona un baremo comparativo con el que calibrar la alzada, peso y volumen de los caballos antiguos. ${ }^{5}$

Hoy en día, un caballo belga, de los más gran- des existentes, puede tener una altura en la cruz de $173 \mathrm{~cm}$., y pesar $1.125 \mathrm{Kg}$. Un percherón puede medir en torno a los $168 \mathrm{~cm}$; un caballo de policía excepcionalmente grande puede llegar a los 182 $\mathrm{cm}$. en la cruz; un caballo de carreras árabe mide hoy en día normalmente entre 147 y $155 \mathrm{~cm}$. Por término medio, los caballos de monta modernos oscilan entre los 142 y los $163 \mathrm{~cm}$. (14-16 manos) de alzada; los de tiro suelen tener entre los $163 \mathrm{y}$ los $173 \mathrm{~cm}$. (16-19 manos). Desde el punto de vista evolucionista, estos tamaños son exagerados, los mayores sólo surgieron a partir del s. XVII, y de hecho suponen una desventaja: son el resultado de la intervención humana a lo largo de siglos (Budiansky, 1997:9 ss. y Fig. 1.2).

En comparación con estos valores, el Tarpan, una raza extinta considerada una de las predecesoras de los caballos actuales, tenía una alzada de en torno a los 120-135 cm., mientras que el Przewalski oscilaba entre los 122 y los $144 \mathrm{~cm}^{6}$

Entre estos dos extremos, los caballos actuales de diversas razas y los predecesores de las estepas euroasiáticas, diferentes estudios vienen mostrando la evolución, por lo general ascendente, de la alzada de los équidos europeos entre la Edad del Bronce y la Edad Media.

El trabajo de H.H. Müller (1993) sobre los caballos de la Edad del Bronce (III-II milenio a.C.) en la Europa central proporciona un valor medio de alzada en la cruz de $125-130 \mathrm{~cm}$.; sólo uno, hallado en Unterhautzenthal, llegaba a los $138 \mathrm{~cm}$. Según uno de los grandes especialistas en la evolución equina, S. Bökönyi (1968: 30 ss. y espec. 39; 1974, 1993), fue precisamente hacia la Edad del Bronce cuando comenzaron a darse grandes divisiones entre los caballos domesticos, que culminaron en que ya en la Edad del Hierro se distinguen dos grupos diferentes... un grupo está formado por los pequeños ponies célticos de Gran Bretaña, Europa Occidentaly Grecia, mientras que el segundo grupo incluye caballos
$4 \mathrm{O}$, por usar la terminología anglosajona, 14 manos (siendo una mano 4 pulgadas, $10.16 \mathrm{~cm}$.). En el terreno deportivo, y según el reglamento aplicado por la Real Federación Hípica Española, un poni es cualquier caballo (no una raza específica): "Un poni es un caballo pequeño cuya alzada, medida en la cruz sobre una superficie llana, no pase de 148 cms. sin herraduras o $149 \mathrm{cms}$. con herraduras (http://82.144.8.107/rfhe/ponis/ponis.htm).

5 Hoy en día se emplean numerosos criterios para clasificar los caballos. La Enciclopedia Británica, por ejemplo, propone clasificaciones por origen "percherón”, “árabe”, "poni Shetland" muchas veces discutido; por estructura ósea y tamaño "ponies" de menos de 14.2 manos, i.e. 144 cm.; "ligeros" de entre 14.2 y 17.2 manos, i.e. $144-175$ cm.; "pesados" de entre 14.2 y 19 manos, i.e., $144-193 \mathrm{~cm}$.; por función (de tiron, de monta...) o por geografía "meridionales" o de "sangre caliente", "septentrionales" o de "san- gre fría" y cruces o "de sangre templada".

6 El Equus ferus ferus (Boddaert 1758) o tarpan, hoy extinto, es objeto de una amplia polémica sobre su posición en la línea evolutiva que llevaría a los caballos actuales. Unos como Nobis (1971:75) creen que el Tarpan de Ucrania estaría en la línea evolutiva; otros lo niegan. El Equus ferus przewalskii (Poliakoff 1881), el caballo salvaje de las estepas mongolas,redescubierto en 1879, es el último representante de los caballos salvajes pleistocenos que en una época habitaron Europa y se vieron empujados hacia las estepas asiáticas (Clutton Brock, 1981:81); también se discute si es ancestro directo o una rama lateral de la génesis del caballo moderno. Entre las síntesis más recientes sobre el origen del caballo domesticado y sus ancestros puede verse el trabajo de Arbogast et al., 2002:17 ss.); tb. Eisenmann (e.p.). 
mayores de Escitia y las estepas rusas (Clutton Brock, 1981:86). Esta división global viene siendo generalmente aceptada (e.g. Audoin-Rouzeau, 1995:81 y Fig. 6; de Grossi et al. 1996:88-89; etc.).

Probablemente los caballos inusitadamente grandes de la Edad del Bronce (II milenio) encontrados en Tebas (Egipto) de $143 \mathrm{~cm}$., y Buhen (Sudán) de $150 \mathrm{~cm}$., o el hitita de Hattusas (Anatolia) de $150 \mathrm{~cm}$. (Hyland, 2003:11; Azzaroli 1996:83) pertenezcan a este grupo (oriental), y hubieran sido considerados bastante grandes incluso en la Roma Imperial, mil quinientos años después; pero son la excepción más que la regla.

\section{LA EdAd del Hierro en Europa.}

S. Bökönyi (1968) publicó un estudio de conjunto de material de Europa centro-oriental, basada incicialmente en la colección de Magdalenska gora de la Primera Edad del Hierro, pero también de kurganes (tumbas monumentales) escitas. Distinguía este autor dos grupos que a grandes rasgos vienen siendo aceptados hasta la actualidad (e.g. Audouin-Rouzeau 1995): el occidental incluye tumbas del Hallstatt de Austria, y La Tène de Alemania y Suiza; el oriental, los Balcanes y Rusia. Según la metodología de determinación de talla empleada, los resultados varían ligeramente, pero bien sea a partir de la longitud de los metacarpos, o por la de los metatarsos, la estimación coincide en que la mayoría de los caballos oscilaba entre los 120 y los $143 \mathrm{~cm}$.

Así, según el análisis de Bökönyi, los caballos del area oriental definida por él eran significativamente mayores que los de la zona occidental. (1968: 30 ss. y espec. 39), aunque el autor (Bökönyi 1968:39) insiste explicitamente en usar la palabra "grupos" y no "raza" o "tipo". Un trabajo posterior, de 1974, precisaba que los caballos "orientales" con la muestra entonces disponible medían entre los 121,9 y los $149.4 \mathrm{~cm}$., con una media de $136,15 \mathrm{~cm}$., mientras que el grupo occidental tenía caballos entre los 109.9 y los $149.4 \mathrm{~cm}$., con una media de sólo 126.07 cm. (Bökönyi 1974:252; 1991:430; Gladitz 1997:129).

Durante la Edad del Hierro en Europa Central y Oriental la mayoría de los caballos medía pues menos de $145 \mathrm{~cm}$. en la cruz, y muchos de ellos serían inferiores a los $126 \mathrm{~cm}$. si la iconografía es fiable, lo que es dudoso (Clutton-Brock, 1981:85); sólo algunos caballos romanos, quizá de origen escita y llegados a Europa a través del mundo macedónico, comenzarían a superar los $145 \mathrm{~cm}$. (Clutton Brock 1981:88; ver infra), pero incluso así, en las estepas euroasiáticas los caballos de 124 a $145 \mathrm{~cm}$. implican una amplia variabilidad, como en los kurganes escitas de Pazyryk (Hyland, 2003:29; Azzaroli, 1985:69). En la zona escita y del
Mar Negro (incluyendo asentamientos griegos y kurganes escitas), durante la Edad del Hierro los caballos más pequeños (de entre 120 y $128 \mathrm{~cm}$. en la cruz) suman el 1.7\% del total; mientras que los mayores $(152-160 \mathrm{~cm}$.) suman sólo el 1.2\% del total. La inmensa mayoría se encuentra pues entre los 128 y los $145 \mathrm{~cm}$. de alzada en la cruz.

Según los estudios morfométricos de Sandor Bókönyi, los caballos de la segunda Edad del Hierro de La Tène en Suiza medirían en torno a los 135.5-141 cm en la cruz (Bökönyi 1968:8, citando a Marek, 1898). Estos antiguos estudios han sido confirmados por trabajos de Liepe (1958) y Franck (1962) sobre los huesos de caballo del oppidum celta de Manching, así como por los posteriores del mismo S. Bököny (1968:8-9). Trabajando sobre diferentes muestras -muy grandes- de materiales, los resultados son similares: los caballos (celtas) de la Edad del Hierro de Manching eran pequeños, de huesos finos, de tipo (occidental), y muy pequeños, con una alzada en la cruz de a los 114 y $137 \mathrm{~cm}$. con sólo algún ejemplar aislado llegando a los 144 y 150 $\mathrm{cm}$. posiblemente de procedencia oriental (Bökönyi 1968, Fig. 15-16).

En Francia, donde los estudios diacrónicos están quizá más avanzados por la amplia muestra disponible, parece claro que desde el Neolítico, durante la Edad del Bronce y hasta la edad del Hierro avanzada (La Tène C2, hacia el 350 a.C.) la talla media de los caballos se mantuvo sobre los $130 \mathrm{~cm}$., para disminuir bruscamente durante los siglos III-I a.C. hasta los $120 \mathrm{~cm}$. -con algunos animales que apenas sobrepasan el metro- por razones desconocidas (Bökönyi sigue a César en opinar simplemente que los galos eran malos criadores de caballos, 1991:430; también 1984:169). Estudios sobre los santuarios de Ribemont sur Ancre, Gournay y Acy-Romance proporcionan recorridos respectivamente entre los 116-136 cm., 116-140 y 108-142, con modas en torno a los $125-130 \mathrm{~cm}$. (Chaix, Meniel 1996:22). La situación no mejoró hasta el cambio de Era, cuando la talla media volvió a aumentar, para superar no sólo los $120 \mathrm{~cm}$. de media anteriores, sino los 130 que habían sido característicos durante milenios, llegando a los 140 $\mathrm{cm}$. en época imperial tardía (Arbogast et al. 2002:46). Meniel (1996:114 ss.) considera que la talla aumentó por la introducción de nuevas razas de origen oriental, con el límite de alzada para diferenciar los dos grupos en torno a los $145 \mathrm{~cm}$., desde La Tène D1 esto es, en torno al 100-50 a.C.

P. Meniel (1987:33 ss; 1996:116) y otros (Arbogast et al. 2002:43-44) distinguen pues entre los caballos galos "nativos" tradicionales, similares al Tarpan, con alzadas de entre 120 y $130 \mathrm{~cm}$., y los caballos "galo-romanos" importados, de origen último oriental, diez centímetros mayores de media, pero con ejemplares que llegan incluso a los 
145 y 156 cm. (Meniel, 1987:37; 1996:116-121 -Montmartin, Beauvais-; Arbogast et al. 2002:45ss.).

Para Italia contamos con otra síntesis basada en datos de unos 85 yacimientos desde el Eneolítico hasta el Bajo Imperio romano (de GrossiMazzorin et al. 1996, alzadas calculadas mediante el coeficiente de May). El caballo eneolítico de Maccarese medía $135 \mathrm{~cm}$.; los de la Edad del Bronce miden de media $128.5 \mathrm{~cm}$. (recorrido: 112 a $142.2 \mathrm{~cm}$.). En la Edad del Hierro los autores distinguen entre caballos 'orientales' mayores y los 'occidentales'. Los primeros están bien representados en el yacimiento paleovéneto de La Brustolede, donde 27 animales adultos fueron sacrificados, algunos todavía con sus bocados. La alzada media de éstos es de 133.8 cm. (recorrido: 117.4 a $146.44 \mathrm{~cm}$.). Los caballos 'occidentales' medidos -en el norte de Italia-presentan una alzada de 123.5 y $127 \mathrm{~cm}$. Sabemos poco de los caballos en Etruria, más al sur, pero los datos disponibles indican una media de $130 \mathrm{~cm}$., algo menor que la de los caballos vénetos. En época romana la alzada media sube a $137.3 \mathrm{~cm}$., llegando a 138.9 en el Bajo Imperio, crecimiento que los autores atribuyen a la creación de una raza mayor por influencia oriental y con destino militar.

\section{EPOCA ROMANA IMPERIAL.}

En efecto, todo indica que la Roma Imperial desarrolló a partir del s. II d.C., por influencia sármata y alana, caballos (militares) de mayor talla (ejemplificados por el caballo de la estatua de Marco Aurelio en Roma, Bökönyi 1984:170; Davis, 1989:33; de Grossi Mazzorin et al. 1996:89), quizá mezclando caballos itálicos nativos con otros orientales de origen escita, según indican fuentes antiguas (Bökönyi 1991:430). De hecho, la arqueología confirma que en Italia exisitieran de antiguo caballos de talla mucho mayor que la habitual en Europa al norte de los Alpes: la tumba céltica de carro del s. III a.C. en Adria (zona véneta de delta del Po), proporcionó los esqueletos bien conservados de tres caballos, dos quizá de tiro y uno de monta (Azaroli 1996). Todos ellos, con alzadas superiores a los $154.4 \mathrm{~cm}$., y $157-158$ en particular para el caballo de tiro, son mayores no sólo que la media, sino superiores a los valores máximos de équidos de Europa Central en la Edad del Hierro.

El gráfico presentado por Audoin-Rouzeau (1995:91, Fig. 6) indica que cuanto más al Sur y Este, mayor es la alzada media de los caballos de época romana imperial (de $136 \mathrm{~cm}$. en Gran Bretaña a $145 \mathrm{~cm}$. en Suiza). Cálculos a partir de huesos hallados en Panonia y otras regiones del proporcionan alturas en la cruz para estos caballos de en torno a los 145-155 cm., sin llegar casi nunca a los 160 cm. (Gladitz 1997:132; Audouin-Rouzeau
1995:82 ; Azzaroli 1985:116; 156).

Por otro lado, el aumento de la talla del caballo doméstico en Europa Occidental durante época romana imperial no es un hecho aislado, sino que se extiende a otras especies como bóvidos, ovicápridos o suidos (Audouin-Rouzeau 1995 passim; Meniel, 1987:22-30)

De todos modos, el mundo antiguo conoció otros caballos de talla "moderna", especialmente en las regiones orientales. Especialmente famosos eran los caballos niseos descritos por Herodoto y otros autores clásicos, considerados especialmente grandes de talla (Azzaroli, 1985:86-89; 176 ss.). Es posible que alguno de estos caballos, según observaciones de Bökönyi, superara los $162 \mathrm{~cm}$. (cit. por Hyland, 2003:30), aunque esto quizá sea excesivo.

\section{EL FIN DEL MUNDo ANTIGUO.}

A fines del mundo antiguo y comienzos de la Edad Media, entre el s. V y el X d.C., en muchas regiones de Europa los caballos apenas llegaban a la alzada de un poni moderno. Así, el hallazgo de un caballo macho robusto en una tumba anglosajona de Sutton Hoo resulta en una alzada en los hombros de $144 \mathrm{~cm}$. "el tamaño de un poni muy grande y robusto" (O־Connor, 1994:31), mientras que los datos comparativos recogidos por este mismo autor en Europa Occidental arrojan que la mayoría de los caballos oscilan entre los 120-140 cm., siendo rarísimos los que llegan a $150 \mathrm{~cm}$. (O'Connor 1994:32 ss.). Una muestra de 107 tumbas Avaras del periodo de las migraciones indica que estos caballos orientales medían de media $134.11 \mathrm{~cm}$., con algunos ejemplares alcanzando los $144.3 \mathrm{~cm}$. (Hyland, 1994:32). En el Oeste, el cementerio belga merovingio de Tournai, fechable entre mediados del s. IV y mediados del VI d.C., el estudio de un total de 21 caballos enterrados en fosas arroja una alzada media para los animales adultos de $142.9 \mathrm{~cm}$., con un recorrido entre los 136.4 y los $156.1 \mathrm{~cm}$., aunque el último caballo es de un tamaño excepcional (Gabriel, Mees, Collin, 115 y 128, Tabla 6). Estos autores recogen valores similares calculados por otros investigadores para caballos de cementerios alamanes contemporáneos o algo posteriores (valores entre 130.5 y 144.7); francos (entre 137.5 y $150.3 \mathrm{~cm}$.) y germanos (entre $135.6 \mathrm{y}$ $141.7 \mathrm{~cm}$.). Los valores medios, en torno a los 140 $\mathrm{cm}$., tienden a enmascarar la presencia todavía de animales muy pequeños, de hasta $123 \mathrm{~cm}$., y de otros ya "modernos" en talla, de hasta $158 \mathrm{~cm}$. (Arbogast et al. 2002:49).

\section{Modelos EXPLICATIVOS}

En síntesis, el panorama global según - parte de- la investigación moderna ha sido resumido por 
M. Teichert del siguiente modo (Teichert 1993:237): En el post-Pleistoceno los caballos salvajes fueron una importante presa de caza para el hombre. Tras su domesticación hacia el 4500 en el Sur de Ucrania los caballos domesticados fueron sólo empleados por su carne. Su empleo como animales de tiro y de monta se hizo popular solo a partir del III y I milenio respectivamente. Los caballos salvajes de Eurasia tenían alturas en la cruz de entre 125 y $135 \mathrm{~cm}$. Tras su domesticación los caballos se hicieron o más pequeños o mayores. En el Imperio Romano las alzadas de los caballos domésticos en Europa Central oscilan entre los 112 y los $145 \mathrm{~cm}$. A partir del periodo de las migraciones [s. Vd.C.] los caballos se hicieron mayores y llegaron a alcanzar los $160 \mathrm{~cm}$. debido a mejores sistemas de cría y alimentación.

Desde el punto de vista diferente de AudoinRouzeau (1995:83) para explicar los mismos datos observados, lo que ocurrió es que los pequeños caballos "occidentales" en la denominación de Bökönyi casi desaparecieron en época imperial romana, salvo en remotas regiones occidentales; pero a su vez los caballos "de guerra" romanos 'hipertrofiados'con una talla de en torno a 145-150 $\mathrm{cm}$. no dejaron descendencia, pues en la época de las migraciones entraron en Europa Occidental grandes cantidades de caballos del tipo "oriental)"de Bökönyi (1974) con tallas algo menores, de entre $135.5 \mathrm{~cm}$. de media: "Los pequeños caballos occidentrales y germánicos de 128 a $130 \mathrm{~cm}$. de talla media desaparecieron de Europa Occidental, reemplazados por una población homogénea de animales de $135.5 \mathrm{~cm}$. de alzada media. La renovación del poblamiento equino de esta Europa es imagen de la renovación de su poblamiento humano. El desplazamiento de ejércitos y pueblos de Este a Oeste implicó el de los caballos orientales hacia Germania. A su vez, estos "nuevos caballos germánicos" fueron trasladados más al oeste. No más que los bóvidos o los corderos, los grandes caballos de los romanos sno parecen haber dejado traza alguna en las antiguas provincias de Roma”. (Audoin-Rouzeau 1995:83).

\section{De los Siglos Oscuros a la Alta Edad MEDIA.}

Durante los siglos Oscuros que siguieron a las invasiones, y durante la Alta Edad Media, el tamaño de los caballos en Europa Occidental fue creciendo de nuevo a causa de las necesidades militares, con el desarrollo de una caballería pesadamente protegida que cargaba al choque con silla arzonada y estribos. Los primeros caballos pesados "de sangre fría" (cold-blooded, en la terminología

7 Para una concienzuda discusión de la iconografía y de algunos textos tardomedievales de los que se deduce que las 'manos' se medían siguiendo el contorno del caballo moderna) aparecieron en Europa Occidental hacia los siglos IX-X d.C. (Gladitz 1997:132; 156 ss.) En regiones occidentales apartadas, como Irlanda, pervivieron durante la Edad Media monturas pequeñas, quizá de tradición gala, como los caballos irlandeses del s.X d.C. de Craigywarren Crannog (Antrim), con alzadas en torno a los $132-142 \mathrm{~cm}$. (Gladitz 1997:145-146). Durante época carolingia en la Galia la media se mantuvo en torno a los 140 cm. (Arbogast et al. 2002:50), pero eso enmascara la existencia de tipos diferentes empleados para necesidades distintas. En concreto, la demanda de destriers, caballos de batalla de gran tamaño, hizo más y más frecuentes animales por encima de los $155 \mathrm{~cm}$., aunque eran excepcionales las alzadas de $160 \mathrm{~cm}$. y más: la variabilidad en tamaños creció considerablemente (Arbogast et al. 2002:46). En Europa Oriental y Asia, sin embargo, la caballería de las estepas seguía prefiriendo monturas ligeras, pequeñas y muy resistentes: por ejemplo, en el s. XIII d.C. los caballos mongoles medían en torno a los 130-135 cm. (Gladitz, 1997:90); en la Rusia medieval la alzada media era de $132.5 \mathrm{~cm}$., con algunos animales llegando a los $152-160 \mathrm{~cm}$. (Gladitz 1997:135).

El reciente estudio de Rackham recuerda que (2004:23) en cierto modo la imagen de los destriers tardomedievales de $170 \mathrm{~cm}$. o más es, desde el punto de vista arqueozoológico, un mito: el gran caballo medieval capaz de cargar un jinete completamente armado existió, pero normalmente no fue mayor de 16 manos $(162.6 \mathrm{~cm} .)^{7}$

\section{Alzada de loS ÉQUidos en Iberia duran- TE LA EdAd dEL HierRo.}

¿Cómo encajan con estos datos que hemos recopilado y ordenado los resultados de los estudios arqueozoológicos sobre la Edad del Hierro en Iberia?. No hay todavía una gran masa de material publicado, dada la escasez de huesos de caballo en los yacimientos ibéricos, y tampoco muchos resultados de síntesis, pero en general puede decirse que son coincidentes. Por ejemplo, el estudio global más reciente sobre una región amplia, el de $\mathrm{P}$. Iborra (2004) sobre el área levantina, incluye el análisis faunístico de los materiales de más de una docena de yacimientos del Bronce Final y sobre todo de la Edad del Hierro valenciano, incluyendo nombres básicos como Vinarragell, el Tossal de San Miguel de Lliria, Castellet de Bernabé. Puntal dels Llops, La Seña, Los Villares de Caudete o la Bastida de les Alcuses. En todo este conjunto, anota

y no en vertical (y que por tanto un "15 manos" de entonces equivaldría a un caballo bastante menor que la misma medida hoy en día) ver Rackham (2004 23 ss.). 
la autora los restos de caballo que hemos identificado son escasos y se encuentran muy fragmentados (Iborra 2004:350), abarcando eso sí niveles desde el Hierro Antiguo al Ibérico Final. Sin embargo, sólo en un caso -en niveles del Bronce Final de Vinarragell- es posible, a través de un metacarpo, calcular una altura en la cruz de $124 \mathrm{~cm}$. En otro trabajo (Iborra 2003:203) añade la autora que calcula que la altura media de los caballos ibéricos levantinos está entre los 120-130 cm. datos plenamente coincidentes con los proporcionados para otros lugares de Europa. Así, una primera revisión de los informes faunísticos publicados en los que se proporciona información sobre alzadas, 8 arroja los siguientes resultados en forma tabular, recogidos aquí:

\begin{tabular}{|c|c|c|c|c|c|}
\hline YACIMIENTO & CRONOLOGIA & ALZADA & METODOLOGIA & CONTEXTO & $\begin{array}{l}\text { BIBLIOGRAFIA } \\
\text { (en negrita la referencia más } \\
\text { precisa) }\end{array}$ \\
\hline $\begin{array}{l}\text { Alcalá de Chivert } \\
\text { (Castellon) }\end{array}$ & $\begin{array}{l}\text { Meds. s. II a.C. } \\
\text { (?) }\end{array}$ & $142 \mathrm{~cm}$. & $\begin{array}{l}\text { Metacarpo tercero. } \\
\text { Factor de Kiesewalter. }\end{array}$ & $\begin{array}{l}\text { Hallado con material } \\
\text { ibérico y campaniense } \\
\text { en una zanja de obra } \\
\text { en Alcalá de Xivert. } \\
\text { ¿Depósito ritual? } \\
\text { Por estado de } \\
\text { conservación, fecha } \\
\text { discutible. }\end{array}$ & $\begin{array}{l}\text { Oliver }(1990,1996) . \\
\text { Iborra (2003) }\end{array}$ \\
\hline $\begin{array}{l}\text { Aldovesta } \\
\text { (Tarragona) }\end{array}$ & $\begin{array}{l}\text { Meds. s. VII } \\
\text { a.C. }\end{array}$ & $136.9 \mathrm{~cm}$. & No especificado & $\begin{array}{l}\text { Poblado preibérico con } \\
\text { materiales fenicios. } \\
\text { Hay además restos de } \\
\text { asno. }\end{array}$ & $\begin{array}{l}\text { Nadal (inéd.); Nadal, } \\
\text { Albizuri (1999:210) }\end{array}$ \\
\hline $\begin{array}{l}\text { Barchín del Hoyo } \\
\text { (Cuenca) }\end{array}$ & s. IV a.C. & $124.9 \mathrm{~cm}$. & $\begin{array}{l}\text { Metacarpo. Indice de } \\
\text { Kiesewalter. }\end{array}$ & $\begin{array}{l}\text { Poblado ibérico. } \\
\text { Campaña } 1979, \\
\text { Cuadricula } 3 \mathrm{~A}^{\prime} \\
\text { Caballo grande en } \\
\text { comparación con otro } \\
\text { de Barchín (I) }\end{array}$ & $\begin{array}{l}\text { Miguel Agueda, Morales } \\
(2002: 67) \\
\text { Sierra Delage (2002) }\end{array}$ \\
\hline & s. IV a.C. (C14) & $\begin{array}{l}\text { ASNO } \\
113,7 \mathrm{~cm}\end{array}$ & $\begin{array}{l}\text { Metacarpiano derecho } \\
\text { de asno adulto. Indice } \\
\text { de Kiesewalter con } \\
\text { factor de corrección } \\
\text { parcial }\end{array}$ & $\begin{array}{l}\text { Poblado ibérico. } \\
\text { Campaña } 1975\end{array}$ & $\begin{array}{l}\text { Morales(1981:230-231) } \\
\text { Castaños (1989:92) }\end{array}$ \\
\hline $\begin{array}{l}\text { Castillar de } \\
\text { Mendavia }\end{array}$ & Hierro I & $\begin{array}{l}124.3 \\
133.25 \\
133.8 \\
137.5\end{array}$ & $\begin{array}{l}\text { Factor de Kiesewalter. } \\
\text { Un metacarpo: } 124.3 \\
\text { cm. } \\
\text { Tres metatarsos: } 133.25 \text {; } \\
133.8 ; 137.5 \mathrm{~cm} .\end{array}$ & $\begin{array}{l}\text { Poblado con un nivel } \\
\text { del Bronce Final y } \\
\text { otros del Hierro I. } \\
\text { Muestra de la segunda } \\
\text { fase, con porcentaje } \\
\text { muy bajo de equidos, } \\
\text { incluyendo posible } \\
\text { asno. }\end{array}$ & Mariezkurrena (1986) \\
\hline $\begin{array}{l}\text { Era Alta (Melgar de } \\
\text { Abajo, Valladolid) }\end{array}$ & $\begin{array}{l}\text { Hierro I? } \\
\text { s. II-I a.C. ? }\end{array}$ & $117 \mathrm{~cm}$ & $\begin{array}{l}\text { No especificada en } \\
\text { Morales, Liesau (1995) } \\
\text { Animal adulto. Fase } \\
\text { cronológica no } \\
\text { especificada }\end{array}$ & $\begin{array}{l}\text { Oppidum vacceo } \\
\text { ampliado en el Hierro } \\
\text { II. La parte excavada } \\
\text { corresponde a un } \\
\text { sector periférico del } \\
\text { oppidum. } \\
\text { La fauna del Hierro I } \\
\text { procede de dos niveles } \\
\text { de habitac. Sucesivos. } \\
\text { La del Hierro II se data } \\
\text { en los ss.II-I a.C. }\end{array}$ & $\begin{array}{l}\text { Morales y Liesau } \\
\text { (1995:481); Romero, } \\
\text { Ramírez (1999); Blasco } \\
\text { Sancho (1999) }\end{array}$ \\
\hline $\begin{array}{l}\text { Hort d'en Grimau } \\
\text { (Barcelona) }\end{array}$ & $\begin{array}{l}\text { Fin s. VII- } \\
\text { principios s. VI } \\
\text { a.C. }\end{array}$ & $\begin{array}{l}118.5 \mathrm{~cm} . \\
\text { (¿asno?) }\end{array}$ & $\begin{array}{l}\text { Factor de Kiesewalter } \\
\text { aplicado a la longitud } \\
\text { lateral del metacarpo } \\
\text { derecho. Los autores } \\
\text { reconocen que el criterio } \\
\text { está pensado para } \\
\text { caballos. Para la } \\
\text { identificación como asno } \\
\text { ver p. } 115 \text {. }\end{array}$ & $\begin{array}{l}\text { Silo E-10 reutilizado } \\
\text { como tumba de } \\
\text { cremación. Encima, } \\
\text { separado por capa de } \\
10-15 \mathrm{~cm} \text {. de piedra y } \\
\text { tierra, équido en } \\
\text { conexión anatómica. }\end{array}$ & $\begin{array}{l}\text { Mestres, Sanmartí, } \\
\text { Santacana (1990). } \\
\text { Albizuri, Nadal (1990) }\end{array}$ \\
\hline La Hoya (Alava) & Hierro II & $\begin{array}{l}128.8 \mathrm{~cm} \\
129 \mathrm{~cm}\end{array}$ & $\begin{array}{l}\text { Metcarapo y metatarso. } \\
\text { Factor de Kiesewalter. } \\
\text { Con el método de Vitt } \\
\text { (1952) se obtienen } \\
\text { resultados de } 1 \text { a } 3 \mathrm{~cm} \text {. } \\
\text { menores. }\end{array}$ & $\begin{array}{l}\text { Poblado. En el } \\
\text { yacimiento también } \\
\text { hay restos de asno, }\end{array}$ & $\begin{array}{l}\text { Altuna (1980:24 ss.;52 y } \\
\text { Tabla 47); Altuna y } \\
\text { Mariezkurrena (1986); } \\
\text { Mariezkurrena (1990); } \\
\text { Liesau (1993, 1998); } \\
\text { Blasco Sancho (1999); } \\
\text { Liesau y Blasco (1999). }\end{array}$ \\
\hline
\end{tabular}

8 Por supuesto, hay numerosos estudios faunísticos -para casi dos centenares de yacimientos de la Edad del Hierro peninsular- que hemos catalogado porque presentan res- tos de équidos, pero o los datos no son suficientes para calcular alzadas o si los hay los autores no presentan ese dato. 


\begin{tabular}{|c|c|c|c|c|c|}
\hline $\begin{array}{l}\text { Muru Astrain } \\
\text { (Sansol, Navarra) }\end{array}$ & $\begin{array}{l}\text { I Edad del } \\
\text { Hierro (Zanja } \\
\text { 25/29, poblado) } \\
\text { Fin II Edad del } \\
\text { Hierro (Zanja I, } \\
\text { necr. } \\
\text { Inhumación) }\end{array}$ & $\begin{array}{l}129 \mathrm{a} \\
134.6 \mathrm{~cm}\end{array}$ & $\begin{array}{l}\text { Restos de } 5 \text { animales. } \\
\text { Estimación para } 2 \text { de } \\
\text { ellos, factor de } \\
\text { Kiesewalter. } \\
\text { Animal 1: Humero: } \\
\text { 129.05; Radio 132.37; } \\
\text { Metacarpo, 134.6. } \\
\text { Animal 2: Metacarpo } \\
\text { 132.04 }\end{array}$ & $\begin{array}{l}\text { Zanja 1: restos de } 3 \\
\text { individuos. Uno de } \\
\text { ellos casi entero con } \\
\text { bocado articulado. } \\
\text { Asociado a tumbas de } \\
\text { inhumación. } \\
\text { ¿cronología posterior } \\
\text { al hierro? El C14 da s. } \\
\text { VII-VII d.C. } \\
\text { Zanja 25/29 (zona de } \\
\text { viviendas): restos de } 2 \\
\text { individuos. }\end{array}$ & $\begin{array}{l}\text { Castaños (1988:225- } \\
\text { 227), Castiella (1990; } \\
\text { 1991-92); Liesau (1993), } \\
\text { Liesau, Blasco (1999) }\end{array}$ \\
\hline $\begin{array}{l}\text { Peñas de Oro } \\
\text { (Alava) }\end{array}$ & $\begin{array}{l}\text { Hierro I Pais } \\
\text { Vasco }\end{array}$ & $\begin{array}{l}130 \mathrm{y} \\
132.2 \mathrm{~cm}\end{array}$ & $\begin{array}{l}\text { Metatarsos. Factor de } \\
\text { Kiesewalter. }\end{array}$ & $\begin{array}{l}\text { Poblado con niveles } \\
\text { desde el Bronce Final } \\
\text { a época romana. Los } \\
\text { restos de caballo son } \\
\text { de la Edad del Hierro. }\end{array}$ & $\begin{array}{l}\text { Altuna (1980:22-23, 52 y } \\
\text { Tabla 47); } \\
\text { Ugartecha et al. (1965, } \\
\text { 1967); Mariezkurrena } \\
\text { (1990); Liesau (1993); } \\
\text { Liesau y Blasco (1999). }\end{array}$ \\
\hline $\begin{array}{l}\text { La Regenta } \\
\text { (Burriana, } \\
\text { Castellón) }\end{array}$ & Ibérica & $\begin{array}{l}136,6 \mathrm{~cm} \\
\text { (Media) }\end{array}$ & $\begin{array}{l}\text { Factor de Kiesewalter. } \\
\text { Media de humero, } \\
\text { cubito, metacarpo III: } \\
136.61 \text {. } \\
\text { Media de Femur, tibia, } \\
\text { metatarso III: } 136.55 \\
\text { Media } 136.63 \text {. } \\
\text { Macho de } 4 \text { años y } \\
\text { medio }\end{array}$ & $\begin{array}{l}\text { Enterramiento de } \\
\text { caballo completo, con } \\
\text { narigón de bronce. }\end{array}$ & $\begin{array}{l}\text { Sarrión (2003a }: 196 ; \\
\text { 2003b). Mesado (2003) }\end{array}$ \\
\hline $\begin{array}{l}\text { Roa de Duero } \\
\text { (Valladolid) }\end{array}$ & $\begin{array}{l}\text { Celtibérico- } \\
\text { Romano }\end{array}$ & $\begin{array}{l}\text { ASNO } \\
108.9\end{array}$ & & & $\begin{array}{l}\text { Castaños (1989:92, } \\
\text { Tabla 6) }\end{array}$ \\
\hline $\begin{array}{l}\text { Soto de Medinilla } \\
\text { (Valladolid) }\end{array}$ & Edad del Hierro & $\begin{array}{l}136,6 \\
\text { (media) }\end{array}$ & $\begin{array}{l}\text { Factor de Kiesewalter. } \\
\text { Radio D: } 137.3 \\
\text { Metacarpo S: } 130.1 \\
\text { Metcarpo S:142 } \\
\text { Media: } 136.6\end{array}$ & $\begin{array}{l}\text { Soto I: Poblado Bronce } \\
\text { Final-Hierro l;cabañas } \\
\text { circulares } \\
\text { Soto II: Hierro I. } \\
\text { Soto III: celtibérico }\end{array}$ & $\begin{array}{l}\text { Liesau (1992) (1993:319); } \\
\text { Morales y Liesau (1995); } \\
\text { Liesau (1998:94-95, } \\
\text { tabla 24); Blasco Sancho } \\
\text { (1999) }\end{array}$ \\
\hline \multirow[t]{2}{*}{ Ubierna (Burgos) } & $\begin{array}{l}\text { II Edad del } \\
\text { Hierro }\end{array}$ & $\begin{array}{l}129,3 \mathrm{~cm} \\
128.8 \mathrm{~cm}\end{array}$ & $\begin{array}{l}\text { Factor de Kiesewalter } \\
\text { Radio: } 129.33 \mathrm{~cm} . \\
\text { Metacarpo: } 128.84 \mathrm{~cm} .\end{array}$ & $\begin{array}{l}\text { Castro Edad del Hierro. } \\
\text { En muestra, restos de } \\
2 \text { adusltos y } 1 \text { juvenil } \\
\text { de menos de } 3,5 \text { años. }\end{array}$ & $\begin{array}{l}\text { Abásolo, Ruiz Vélez } \\
\text { (1979); Castaños } \\
\text { (1989:90,y Tabla 3); } \\
\text { Liesau (1993); Liesau } \\
\text { (1998) }\end{array}$ \\
\hline & & $\begin{array}{l}\text { ASNO } \\
102.3 \mathrm{~cm} .\end{array}$ & $\begin{array}{l}\text { Factor de Kiesewalter. } \\
\text { No se especifica factor } \\
\text { corrector. } \\
\text { Metatarso }\end{array}$ & & \\
\hline $\begin{array}{l}\text { Els Vilars (Arbeca, } \\
\text { Lérida) }\end{array}$ & $\begin{array}{l}\text { Ibérico antiguo } \\
\text { c. } 550-\text {-c. } 425 \\
\text { a.C. }\end{array}$ & $\begin{array}{l}115-116 \\
\mathrm{~cm} . \text { (poni o } \\
\text { asno) }\end{array}$ & $\begin{array}{l}\text { Factor no especificado } \\
\text { Alzada aproximada } \\
\text { Metatarso; fragmento } \\
\text { distal de tibia }\end{array}$ & $\begin{array}{l}\text { Poblado fortificado con } \\
\text { varias fases. El equido } \\
\text { pertenece a la fase } \\
\text { Vilars II. }\end{array}$ & $\begin{array}{l}\text { Alonso et al. (1994- } \\
\text { 1996:); Gómez Flix } \\
\text { (2003); Belarte, Sanmarti } \\
\text { (1997); Franquesa et al. } \\
\text { (2000); Vilars (1997) }\end{array}$ \\
\hline $\begin{array}{l}\text { Villasviejas de } \\
\text { Botija (Cáceres) }\end{array}$ & s. IV-II a.C. & $\begin{array}{l}\text { Unos } \\
130 \mathrm{~cm} .\end{array}$ & $\begin{array}{l}\text { Factor no especificado } \\
\text { Metacarpiano }\end{array}$ & $\begin{array}{l}\text { Poblado. Muestra } \\
\text { analizada de niveles I y } \\
11.7 \text { restos de } 4 \\
\text { individuos. }\end{array}$ & $\begin{array}{l}\text { Bustos et al. (1989:147); } \\
\text { Hernández et al. (1989) } \\
\text { (1989); Castaños (1991, } \\
\text { 1998). }\end{array}$ \\
\hline
\end{tabular}

Figura 1. Alzada de équidos de la Edad del Hierro peninsulas

Como se puede apreciar, la muestra es demasiado escasa y dispersa en el tiempo y en el espacio -con predominio de la zona norte peninsularcomo para que el valor medio $(132 \mathrm{~cm}$., recorrido $117-137,5)^{9}$ sea de mucha utilidad real. ${ }^{10}$ Sin embargo el conjunto de valores (Figuras 1 y 2 ) encajan perfectamente dentro de los que hemos visto como comunes en la Edad del Hierro de Europa Central,

9 Dejando aparte el caso discutible de Alcalá de Chivert (moderno?) y los posibles asnos.

10 Altuna (1980:52) proporciona para cuatro casos de la en su variante 'occidental' de Bokönyi (1974:252).

Por orden aproximadamente cronológico, los valores siguen esta dispersión (conscientemente no presentamos gráfico por periodos dada la escasez de la muestra) : s. VII a.C., $136.9 \mathrm{~cm}$. (Aldovesta); ss.VII-VI a.C.: $118.5 \mathrm{~cm}$. (Hort d'en Grimau, posible asno); s. V-IV a.C.: 125, 115, 130 cm.; s. II a.C.: $142,117 \mathrm{~cm}$. El resto presentan dataciones dema-

Edad del Hierro en el País Vasco alzadas entre 128.8y 132; Mariezkurrena (1990:250) proporciona para ocho casos alzadas de entre 124.3 y $137.5 \mathrm{~cm}$. en un periodo mayor. 


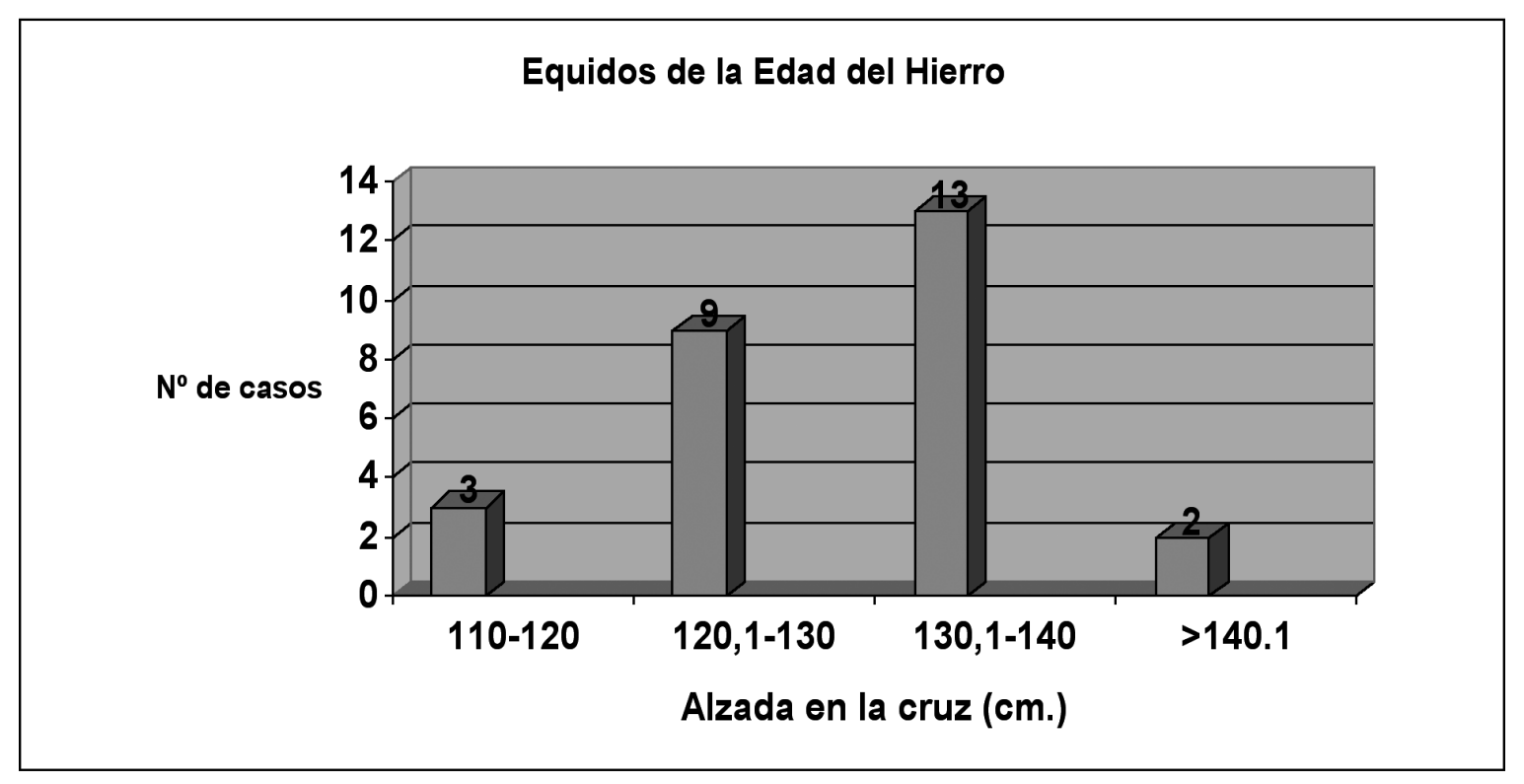

Figura 2. Alzada de équidos de la Edad del Hierro en Iberia

siado genéricas. Como se ve, no hay un patrón claro, pero esto era de esperar dadas las reducidas dimensiones de la muestra. Separar los pocos datos disponibles entre la Primera y la Segunda Edad del Hierro será imprescindible en el futuro, cuando la serie de datos sea mayor, dada la presumible introducción de nuevas razas y selección de animales; los datos son simplemente demasiado escasos por ahora como para que sean estadisticamente significativos.

En conjunto, resulta claro que nos movemos en valores normales en términos europeos, pero también parece evidente que es necesario un esfuerzo sistemático de medición de los -por otra parte porcentualmente muy escasos- restos de équidos en yacimientos de la Edad del Hierro para contar con series aceptables. Esto choca con la evidencia de que en los casi doscientos yacimientos de la Segunda Edad del Hierro con análisis de fauna que hemos catalogado en nuestra base de Datos $\mathrm{SGDE}^{11}$ rara vez el porcentaje de restos de équido sobrepasa el 5\%, siendo normales cifras incluso del $1 \%$. En tales condiciones es difícil encontrar una buena serie para establecer mediciones al estilo de algunas realizadas en oppida o santuarios celtas.

\section{EN TORNO A LA UTILIDAD DE LOS CABALLOS DE PEQUEÑa TALLA.}

Sabemos pues que los caballos antiguos de la Europa Occidental-ibéricos incluidos- eran, incluso en época imperial romana, más pequeños incluso que muchos poneis actuales. Debemos de ello deducir que eran demasiado pequeños y débiles para ser montados?. La respuesta es que no. Aparte de la masa de información iconográfica y sobre todo literaria que nos documenta directa y paladinamente la resistencia de estos pequeños caballos antiguos, sabemos por datos modernos que, contrariamente al mito general, los caballos pequeños son especialmente resistentes y no demasiado débiles de espalda para la monta (Hyland, 1994:172, n. 34). Katryhyn Beck escribía en el foro especializado de arqueología Aegeanet el 20 de Marzo de 1998: Mi poni, que montaba durante millas todos los días, era mitad poni local y mitad caballo de silla australiano y con sus 13.5 manos -unos $137 \mathrm{~cm}$-- era el caballo más grande de la isla de Sumatra. Los poneys locales tenían entre 10 y 12.5 manos -101.6 a 127 cm.- y llevaban cómodamente a jóvenes todo el día al trote. Podían también así cargados mostrar una punta impresionante de velocidad como para acomodarse a la proverbial carga de caballería. Ann Hyland, experta jinete ganadora de diversos concursos de resistencia equestre, insiste en que ni la velocidad ni el tamaño son decisivos en un caballo de guerra, mientras que su conformacion sí que lo es (1990:67). Cualquier caballo tiene la suficiente punta de velocidad para la carga de caballería normal. Lo importante es que cuanto más compacto es el animal, mayor su capacidad para llevar carga (Hyland, 1990:67). Sin duda en campaña el criterio fundamental para un caballo era su resistencia a malos forrajes y a problemas intestinales, $y$ una complexión resistente más que el tamaño o la velocidad punta (Hyland, 1990:69-70; 122 ss.).

JINETES E IMÁGENES.

11 Ver http://www.ffil.uam.es/equus 
No es esta breve nota lugar para entrar en la cuestión de la talla y peso de los jinetes de la Edad del Hierro, pero aunque en general los datos disponibles indican una talla media menor para la población humana que en la actualidad -no podemos disponer de datos para las tumbas de cremación ibéricas de la Edad del Hierro-, también es cierto que en la Primera Edad del Hierro europea los aristócratas que montaban a caballo -el carro de guerra no estaba extendido en este periodo, $c f$. Quesada 2005:56 ss.) tenían estaturas a menudo incluso superiores que la media actual (Hochdorf $187 \mathrm{~cm}$.; Asperg $184 \mathrm{~cm}$.) mientras que en el s. VI a.C. la media de los cementerios en Wurtemberg arroja una media de $172 \mathrm{~cm}$. similar a la actual de Francia (Brun 1987:97 ss.), y la caballería romana imperial contaba con jinetes altos y corpulentos, incluso superiores a los $180 \mathrm{~cm}$. (Speidel 1994:77; Vegecio Epit. Rei Milit., 1.5); por otro lado, los poneis modernos como se ha dicho cargan adultos sin dificultad cuando su conformación es correcta.

Finalmente, no intentaremos siquiera utilizar la iconografía para tratar de determinar la talla de los animales, ni en el mundo del arte peninsular, y ni siquiera en el mejor arte clásico. Las distorsiones de escala por razones iconográficas son demasiado grandes para que tal aproximación tenga alguna utilidad (e.g. Azzaroli 1996:84).

\section{Bibliografía}

ABASOLO, J.A.; RUIZ VELEZ, I. (1979) "El conjunto arqueológico de Ubierna. Contribución al estudio de la Edad del Hierro en la Meseta Norte". BSEAA 45, pp. 168-188.

ALBIZURI, S.; NADAL, J. (1990) "Estudi de l'èquid aparegut en relació amb l'estructura E10 de L'Hort d'en Grimau”. Olerdulae 15, 1-4, pp.112-117.

ALONSO MARTINEZ, N. et alii (1994-96) "L'assentament dels Vilars (Arbeca, les Garrigues):territori, recursos i activitats productives". J. Rovira (ed.), Models d'ocupació..., Gala 3-5, pp. 319-340.

ALTUNA, J. (1980) Historia de la domesticación animal en el Pais Vasco. Desde sus orígenes a la Romanización. Munibe 32, pp. 1-163.

ALTUNA, J.; MARIEZKURRENA, K. (1986) "Introduction de l'Ane (Equus Asinus) au Pays Basque". Archaeozoologia (Mélanges à l'occasion du 5e Congrès intern. d'archéozoologie, Grenoble, pp. 125-130.

ARBOGAST, R.M.; CLAVEL, B.; LEPETZ, S.; MENIEL, P.; YVINEC, J.H. (2002) Archéologie du cheval. Des origines à la période moderne en France. Paris.
AUDOIN-ROUZEAU, F. (1995) "La taille des animaux d'élevage a l'époque romaine et leur exportation". En Homme et animal dans l'Antiquité Romaine. Actes du Colloque de Nantes 1991. Caesarodunum, $n^{0}$ hors serie. Tours, pp. 79-100.

ANREITER, P.; BARTOSIEWICZ, L.; JEREM, E.; MEID, W. (eds.) (1998) Man and the Animal World. Studies in Archaeozoology, Archaeology, Anthropology and Palaeolinguistics in memoriam Sándor Bökönyi. Budapest.

AZZAROLI, A. (1985) An Early History of Horsemanship. Leiden.

AZZAROLI, A. (1996) "The Canal Bianco Tomb of Adria, and large sized horses in the Ancient World". En Proceedings of the XIII Congress of the International Union of Prehistoric and Protohistoric Sciences, Forli, 1996; Vol. 6.I, pp. 83-85.

BELARTE FRANCO, M.C.; SANMARTI, J. (1997) "Espais de culte i pràctiques rituals a la Catalunya protohistòrica". Espacios y lugares cultuales en el mundo ibérico, CPAC 18, pp. 7-32.

BLASCO SANCHO, M.F. (1999) "Factores condicionantes de la composición de la cabaña ganadera de la II Edad del Hierro en la mitad norte de la Península Ibérica". En F. Burillo (ed.) IV Simposio sobre Celtíberos. Economía. Zaragoza, pp. 149-156.

BODSON, L. (1995) Le cheval et les autres áquides: aspects de l'histoire de leur insertion dans les activités humaines. Colloques d'histoire des connaissances zoologiques, 6 . Liège.

BOESSNECK, J. et al. (1971) "Die Tierknochenfunde aus dem Oppidum von Manching". En Die Ausgrabungen in Manching, 6. Wiesbaden.

BÖKÖNYI, S. (1968) Data on Iron Age Horses of Central and Eastern Europe. Cambridge, Mass.

BÖKÖNYI, S. (1974) History of domestic mammals in Central and Eastern Europe. Budapest.

BÖKÖNYI, S. (1984) “Horse”. En I.L. Mason (ed.) Evolution of domesticated animals. London\&New York, 162-173.

BÖKÖNYI, S. (1991) "L'allevamento". En I Celti. Milano, pp. 429-435.

BÖKÖNYI, S. (1993) Pferdedomestikation, Haustierhaltung und Ernährung. Archaeolojguia Series Minor, 3. Budapest.

BRUN, P. (1987) Princes et princesses de la Celtique. Le premier Age du Fer (850-450 av. J.-C.) Paris.

BUDIANSKY, S. (1997) The nature of Horses. Their evolution, intelligence and behaviour. London.

BUSTOS PRETEL, V.; MOLERO, G.; BREA, P. (1989) "Estudio faunístico del yacimiento de Villasviejas (Botija, Cáceres). En F. Hernández et al. Excavaciones en el castro de Villasviejas... Mérida, pp. 143-164. 
CASTAÑOS UGARTE, P.M. (1986) "Restos óseos de $L a$ Cava”. J.D. Sacristán de Lama, La Edad del Hierro en el valle medio del Duero. Valladolid, pp. 275-285.

CASTAÑOS UGARTE, P.M. (1988) "Estudio de los restos óseos de Muru-Astrain". Trabajos de Arqueología Navarra 7, pp. 221-235.

CASTAÑOS UGARTE, P. (1989) "Estudio de los restos óseos del castro de Ubierna (Burgos)”. Kobie 18, pp. 87-97.

CASTAÑOS UGARTE, P.M. (1991) "Animales domésticos y salvajes en Extremadura. Origen y evolución". Rev. de Estudios Extremeños 47.1, pp. 9-66.

CASTAÑOS UGARTE, P.M. (1998) "Evolución de las faunas protohistóricas en Extremadura". En A. Rodríguez Díaz (ed.). Extremadura protohistórica:paleoambiente, economía... Cáceres, pp. 63-72.

CASTIELLA, A. (1990) "Enterramientos en el contexto protohistórico de Sansol (Muru-Astrain, Navarra)". F. Burillo (ed.) II Simposio sobre los celtíberos. Necrópolis Celtibéricas. Zaragoza, pp. 149-157.

CASTIELLA, A. (1991-92) "Cosnideraciones sobre el poblado y necrópolis de Sansol (Muru Astrain, Navarra)". Trabajos de Arqueología Navarra 10, pp. 225-286.

CHAIX, L.; MENIEL, P. (1996) Éléments d'archéozoologie. Paris.

CLARK, J. (2004) (ed.) The Medieval horse and its equipment. Museum of London.

CLASON, A.; PAYNE, S.; UERPMANN, H.P. (1993) Skeletons in her cupboard. Festschrift for Juliet CluttonBrock. Oxbow monographs 34, Oxford.

CLUTTON-BROCK, J. (1981) Domesticated animals from early times. London.

CLUTTON-BROCK, J. (1992) Horse power. A history of the horse and the donkey in human societies. London.

DAVIS, R.H.C. (1989) The Medieval Warhorse. Origin, development and redevelopment. London.

DE GROSSI MAZZORIN, J.; RIEDEL, A.; TAGLIACOZZO, A. (1996) "Horse remians in Italy from the Eneolithic to the Roman Period". En Proceedings of the XIII Congress of the International Union of Prehistoric and Protohistoric Sciences, Forli, 1996; Vol. 6.I, pp. 87-92.

EISENMANN, V. (1996) "Quaternary Horses: possible candidates to domestication". En Proceedings of the XIII Congress of the International Union of Prehistoric and Protohistoric Sciences, Forli, 1996; Vol. 6.I, pp. 27-36.

EISENMANN, V. (e.p.) "What kind of horse at Botai?". En A. Gardeisen, A. Hermary (eds.) Les equides dans le monde méditerraneen antique. Colloque Athènes, Novembre 2003.

FRANCK, K.G. (1962) "Neue funde des pferdes aus dem keltischen Oppidum von Manching". Studien an vor- und frühgeschichtlichen Tierresten Bayerns, XIII. München.

FRANQUESA CASACUBERTA, D.; OLTRA, J.; PIÑA, A.; PONS, E. (2000) "La ramadería en les societats ibèriques del N_E de la Península Ibèrica: diversificació i especialització". En C. Mata, G. Pérez (eds.) Ibers. Agricultors, artesans i comerciants, Saguntum Extra 3, pp. 153-161.

GABRIEL, A.; MEES, G.; COLLIN, B. (1995) "Ëtude morphométrique des squelettes équins mérovingiens de Tournai". En L. Bodson (ed.) Le cheval et les autres équidés: aspects de l'histoire de leur insertion dans les activités humaines. Journée d'étude Université de Liège, 12 mars 1994. Liège, pp.103-131.

GAUTIER, A. (1996) 'The 'unacceptable face' of the Western European Palaeolithic revisited: the evidence for the presumed domestication of the horse during that period". En Proceedings of the XIII Congress of the International Union of Prehistoric and Protohistoric Sciences, Forli, 1996; Vol. 6.I, pp. 45-50.

GHEORGHIOU, D. (1996) "Evidence of horse domestication in $5^{\text {th }}-4^{\text {th }}$ millennia $\mathrm{BC}$ Eastern Europe". En Proceedings of the XIII Congress of the International Union of Prehistoric and Protohistoric Sciences, Forli, 1996; Vol. 6.I, pp. 101-106.

GLADITZ, Ch. (1997) Horse breeding in the Medieval World. Dublin.

GOMEZ FLIX, X. (2003) "Fetos de équido en Els Vilars (Arbeca, Lleida). Un nuevo tipo de deposición animal en hábitat durante la Primera Edad del Hierro en Cataluña". En F. Quesada, M. Zamora (eds.) El caballo en la antigua Iberia. Madrid, pp. 209-217.

HERNANDEZ HERNANDEZ, F.; RODRIGUEZ, M.D.; SANCHEZ, M.A. (1989) Excavaciones en el Castro de Villasviejas del Tamuja (Botija, Cáceres). Mérida.

HOMME ET ANIMAL (1995) Homme et animal dans l'Antiquité romaine. Actes du Colloque de Nantes 1991. Caesarodunum, $\mathrm{n}^{\mathrm{o}}$ hors serie. Tours.

HYLAND, A. (1990) Equus. The Horse in the Roman World. London.

HYLAND, A. (1994) The Medieval Warhorse. From Byzantium to the Crusades. Stroud.

HYLAND, A. (2003) The horse in the Ancient World. Stroud.

IBORRA, P. (2003) "Equidos y restos faunísticos de la Calle San José de Alcalá de Xivert (Castellón)". En F. Quesada, M. Zamora (eds.) El caballo en la antigua Iberia. Madrid, pp. 201-208.

IBORRA, P. (2004) La ganadería y la caza desde el Bronce Final hasta el ibérico final en el territorio valenciano. SIP Trabajos Varios 103. Valencia.

LEVINE, M. A. (1993) "Social Evolution and Horse Domestication". En C. Scarre, F. Healy (eds.) Trade and 
exchange in Prehistoric Europe. Proceedings of a Conference held at the University of Bristol. April 1992. Oxford, pp. 135-141.

LEVINE, M. A.(2004) "Exploring the criteria for Early Horse domestication". M. Jones (ed.) Traces of Ancestry: studies in honour of Colin Renfrew. Cambridge, pp. 115-126.

LEVINE, M.; RENFREW, C.; BOYLE, K. (eds.) (2003) Prehistoric steppe adaptation and the horse. Cambridge.

LIEPE, H.U. (1958) "Die Pferde des latène Oppidums Manching". Studien an vor- und frühgeschichtlichen Tierresten Bayerns, IV. München.

LIESAU VON LETTOW-VORBECK, C. (1993, inéd.) Contribución al estudio arqueofaunístico durante la Edad del Hierro en la Submeseta norte de la Península Ibérica. Tesis doctoral Universidad Autónoma de Madrid. Microficjhas ISBN no ${ }^{\circ}$ 14-7477-466-7.

LIESAU VON LETTOW-VORBECK, C. (1998) El Soto de Medinilla: faunas de mamíferos de la Edad del Hierro en el Valle del Duero (Valladolid, España). Archaeofauna 7. Salamanca.

LIESAU VON LETTOW-VORBECK, C. (2005) "Arqueozoología del caballo en la antigua Iberia". En M. Barril, F. Quesada (eds.) El Caballo en la Antigüedad. Madrid.

LIESAU VON LETTOW-VORBECK, C.; BLASCO, C. (1999) "Ganadería y aprovechamiento animal". En F. Burillo (ed.) IV Simposio sobre Celtíberos. Economía. Zaragoza, pp. 119-147.

MARIEZKURRENA, K. (1986) "La cabaña ganadera del Castillar de Mendavia (Navarra). Munibe 38, 119-169.

MARIEZKURRENA，K. (1990) "Caza y domesticación durante el Neolítico y Edad de los Metales en el País Vasco". Munibe 42, pp. 241-252.

MEADOW, R.H.; UERPMANN, H.P. (eds.) (1991) Equids in the Ancient World II. Wiersbaden.

MENIEL, P. (1987) Chasse et elevage chez les Gaulois (450-52 av. J.C.). Paris.

MENIEL, P. (1990) "Modifications de l'elevage a la fin de l'Age du Fer". Rev. d'Archéologie de l'Ouest. Suppl. 3, pp. 271-276.

MENIEL, P. (1996) "Importation de grands animaux romains et amélioration du cheptel à la fin de l'Âge du Fer en Gaule Belgique". Revue Archéologique de Picardie, 3-4, pp. 113-122.

MESADO, N. (2003) "El caballo ibérico de La Regenta (Burriana, Castellón)". En En F. Quesada, M. Zamora (eds.) El caballo en la antigua Iberia. Madrid, pp. 179186.

MESTRES, J.; SANMARTI, J.; SANTACANA，J. (1990) "Estructures de la Primera Edat del Ferro de l'Hort d'en Grimau (Castellví de la Marca, Alt Penedès)". Olerdulae 15, pp. 75-117.
MIGUEL AGUEDA, J.; MORALES, A. (2002) "Segundo informe sobre los restos faunísticos de vertebrados recuperados en el yacimiento 'Guente la Mota' (Barchín del Hoyo)". En M. Sierra, Yacimiento ibérico Fuente la Mota, pp. 33-71.

MORALES MUÑIZ, A. (1981) "Primer informe sobre la fauna de Barchín”. En Sierra Delage, 1981, pp. 227236.

MORALES MUÑIZ, A. (1988) "Identificación e identificabilidad: cuestiones básicas de metodología arqueozoológica”. Espacio, Tiempo y Forma, Serie I, Prehistoria, 1, pp. 455-470.

MORALES MUÑIZ, A. (1990) "Arqueozoología teórica: usos y abusos reflejados en la interpretación de las asociaciones de fauna de yacimientos antrópicos". TP 47, pp. 251-290.

MORALES MUÑIZ, A.; LIESSAU, C. (1995) "Análisis comparado de las faunas arqueológicas en el valle medio del Duero (prov. Valladolid) durante la Edad del Hierro". Arqueología y Medio ambiente.El 1er milenio a.C. en el Duero, pp. 455-514. Valladolid.

MORALES MUÑIZ, A. et alii (1996) "A prelliminary catalogue of Holocene equids in the Iberian Peninsula". En Proceedings of the XIII Congress of the International Union of Prehistoric and Protohistoric Sciences, Forli, 1996; Vol. 6.I, pp. 65-82.

MÜLLER, H.H. (1993) "Horse skeletons of the Bronze Age in Central Europe". En A. Clason, S. Payne, H. Uerpmann (eds.), Skeletons in her Cupboard. Festschrift Clutton-Brock. Oxford, págs. 143-150.

NADAL LORENZO, J. (Inéd.) "Dades preliminars sobre les restes d'èquids apareguts al jaciment d'Aldovesta".

NADAL LORENZO, J.; ALBIZURI, S. (1999) "El Barranc de Gàfols (Ginestar, Tarragona) y Aldovesta (Benifallet, Tarragona): el estudio arqueozoológico como base de teorización sobre la dieta humana..." Pyrenae 30, pp. 207-221.

O'CONNOR, T. (1994) "A horse skeleton from Sutton Hoo, Suffolk, U.K.". Archaeozoologie 7.1, pp. 29-37.

OLIVER FOIX, A. (1990) "Hallazgo arqueológico en el casco urbano de Alcalá de Xivert". Boletín del Centro de Estudios del Maestrazgo 40, pp. 81-88.

OLIVER FOIX, A. (1996) "Fauna y vegetación en los ritos cultuales ibéricos”. CPAC 17, pp. 281-308.

QUESADA, F. (2005) "Carros en el antiguo Mediterráneo: de los orígenes a Roma”. E. Galán (Ed.) Historia del Carruaje en España. Madrid, pp. 16-71.

RACKHAM, J. (2004) "Physical remains of medieval horses.The size of the medieval horse”. En J. Clark (ed.) The Medieval horse.... pp.19-32. 
ROMERO CARNICERO, F.; RAMIREZ RAMIREZ, M.L. (1999) "Estrategias de subsistencia en la Cuenca Media del Duero durante la Edad del Hierro". En F. Burillo (ed.), IV Simposio sobre Celtíberos. Economía. Zaragoza, pp. 453-465.

SARRION, I. (2003a) "Osteometría del caballo ibérico de La Regenta” (Burriana). En En F. Quesada, M. Zamora (eds.) El caballo en la antigua Iberia. Madrid, pp. 191-200.

SARRION, I. (2003b) "El caballo de La Regente (Nurriana). Estado de conservación y restauración". En F. Quesada, M. Zamora (eds.) El caballo en la antigua Iberia. Madrid, pp. 187-190.

SIERRA DELAGE, M. (1981) "Fuente de la Mota (Barchín del Hoyo, Cuenca)". NAH 11, pp. 209-306.

SIERRA DELAGE, M. (2002) Yacimiento ibérico 'Fuente la Mota' (Barchín del Hoyo, Cuenca). Cuenca.

SPEIDEL, M.P. (1994) Riding for Caesar. The Roman emperor's Horse Guard. London.

TEICHERT, M. (1993) "Size and utilisation of the most important domesticated animals in Central Europe from the beginning of domestication until the late middle ages". En A. Clason, S. Payne, H. Uerpmann (eds.), Skeletons in her Cupboard. Fest. Clutton-Brock, pp. 235-238. Oxford.

UERPMANN, H.P. (1990) "Die domestikation des pferdes im Chalkolitikum West- und Mitteleuropas". Madrider Mitteilungen 31, pp. 109-153.

UERPMANN, H.P. (1995) "Domestication of the HorseWhen, Where and Why?". En L. Bodson (ed.) Le cheval et les autres équidés: aspects de l'histoire de leur insertion dans les activités humaines. Journée d'étude Université de Liège, 12 mars 1994. Liège, pp. 15-29.

UGARTECHA, J.M. et alii (1965) "El Castro de las Peñas de Oro (Valle de Zuya, Alava)". Boletín de la Institución Sancho el Sabio, 11, pp 121 ss.

UGARTECHA, J.M. et alii (1967) "El Castro de las Peñas de Oro (Valle de Zuya, Alava)". Boletín de la Institución Sancho el Sabio, 13, pp 209-220.

VILARS 2000 (1997) Vilars 2000. Una fortalesa ilergeta d'ara fa 2.700 ans. Lleida. 